\title{
Optic Nerve Crush: Axonal Responses in Wild-Type and bcl-2 Transgenic Mice
}

\author{
Sabrina Chierzi, ${ }^{1,2}$ Enrica Strettoi, ${ }^{1}$ Maria Cristina Cenni, ${ }^{1}$ and Lamberto Maffei ${ }^{1,2}$ \\ Istituto di Neurofisiologia del Consiglio Nazionale delle Ricerche, and '2Scuola Normale Superiore, 56127 Pisa, Italy
}

Retinal ganglion cells of transgenic mice overexpressing the anti-apoptotic protein $\mathrm{Bcl}-2$ in neurons show a dramatic increase of survival rate after axotomy. We used this experimental system to test the regenerative potentials of central neurons after reduction of nonpermissive environmental factors. Survival of retinal ganglion cells 1 month after intracranial crush of the optic nerve was found to be $100 \%$ in adult bcl- 2 mice and $44 \%$ in matched wild-type (wt) mice. In the optic nerve, and particularly at the crush site, fibers regrowing spontaneously or simply sprouting were absent in both wt and bcl-2 mice. We attempted to stimulate regeneration implanting in the crushed nerves hybridoma cells secreting antibodies that neutralize central myelin proteins, shown to inhibit regeneration (IN-1 antibodies) (Caroni and Schwab, 1988). Again, we found that regeneration of fibers beyond the site of crush was virtually absent in the optic nerves of both wt and bcl-2 mice. However, in bcl-2 animals treated with IN-1 antibodies, fibers showed sprouting in the proximity of the hybridoma implant. These results suggest that neurons overexpressing bcl-2 are capable of surviving axotomy and sprout when faced with an environment in which inhibition of regeneration has been reduced. Nevertheless, extensive regeneration does not occur, possibly because other factors act by preventing it.

Key words: survival; axotomy; regeneration; sprouting; bcl-2; myelin
In the adult CNS of mammals, regeneration of transected axons is usually absent. The main reasons are usually ascribed to the massive death of damaged neurons and to the inhibitory role exerted by CNS.

The optic nerve is a suitable CNS model to approach the problem of regeneration. Its transection leads to the fast and massive death of axotomized retinal ganglion cells (RGCs) (Villegas-Perez et al., 1988; Berkelaar et al., 1994). Several strategies have been followed to increase their survival (Carmignoto et al., 1989; Thanos et al., 1989; Maffei et al., 1990; MansourRobaey et al., 1994); recently, we showed the protecting effects of the anti-apoptotic gene bcl-2 on axotomized RGCs (Bonfanti et al., 1996; Cenni et al., 1996).

The upregulated expression of bcl-2 prevents neuronal death in several in vitro systems (Allsopp et al., 1993; Zhong et al., 1993). The efficacy of bcl-2 in promoting neuronal survival in vivo has been evaluated by means of a transgenic mouse overexpressing the bcl-2 gene in neurons (Martinou et al., 1994); 2 months after the transection of the optic nerve, $63 \%$ of retinal ganglion cells survive in the bcl-2 mouse, whereas survival in the wild type (wt) is $5 \%$. The proximal segments of injured axons, at least near to the optic nerve head, is also preserved (Cenni et al., 1996); light responses in RGCs are maintained as well (Porciatti et al., 1996). Among the numerous attempts to counteract the process of

Received Dec. 21, 1998; revised July 14, 1999; accepted July 16, 1999.

This work was partially supported by the European Economic Community (Biotech Grant BIO4-CT96-0774) and by the TeleThon Foundation Project 934. We are indebted to Dr. Martin E. Schwab for the IN-1 and HRP hybridoma cells. We thank Dr. GianMichele Ratto and Dr. Tommaso Pizzorusso for helpful comments on this manuscript; Dr. Elena Putignano for performing PCR; and the staff of the Istituto di Neurofisiologia for technical support.

Drs. Chierzi and Strettoi contributed equally to this work.

Correspondence should be addressed to Enrica Strettoi, Istituto di Neurofisiologia del Consiglio Nazionale delle Ricerche, Via San Zeno 51, 56127 Pisa, Italy.

Copyright (C) 1999 Society for Neuroscience $0270-6474 / 99 / 198367-10 \$ 05.00 / 0$ ganglion cell death after optic nerve lesion, bcl-2 overexpression is by far the most successful strategy.

We asked whether axotomized RGCs of bcl-2 transgenic mice are able to regrow their severed fibers in vivo across the lesion site. This question seemed particularly relevant in view of recent findings supporting the notion that the bcl-2 gene is implicated in neuronal differentiation (Middleton et al., 1998), rate of axonal elongation (Hilton et al., 1997), and duration of cell cycle steps (Adams and Cory, 1998). In addition, it has been reported that cultured RGCs from adult bcl-2 mice retain the ability of growing their processes on embryonic tectal slices (Chen et al., 1997).

To study regeneration in the optic nerve, the strong hostility of mature CNS to axonal elongation has to be counteracted. Experiments in vivo show that mammalian RGCs are indeed capable of regenerating their axons through transplants of peripheral nerves (Vidal-Sanz et al., 1987). Inhibitory molecules have been identified that arrest growth cone progression; in particular, a $250 \mathrm{kDa}$ protein fraction, purified from CNS myelin, exerts a powerful antagonistic effect on axonal growth; the monoclonal antibody IN-1, raised against this fraction (Caroni and Schwab, 1988), is capable of effectively counteracting the inhibitory action of CNS myelin, allowing neuronal regeneration in many different CNS areas (for review, see Brösamle and Schwab, 1996).

In this study, we made use of the IN-1 antibody to create a less hostile environment for fiber regrowth; we crushed the optic nerve of wt and bcl-2 mice and implanted hybridoma cells secreting the IN-1 antibody in the proximity of the damaged nerve. We report the effects of such treatment 1 month after crush.

\section{MATERIALS AND METHODS}

Animals. Transgenic mice from the NSE73a line, developed by J-C Martinou (Serono, Geneva, Switzerland), were used in the present study as in previous studies (Cenni et al., 1996; Chierzi et al., 1998). These mice overexpress the human protein $\mathrm{Bcl}-2$ in neurons under the control of neuron-specific enolase. Characteristics of the transgenics are re- 
ported in Martinou et al. (1994). Briefly, our colony started in 1994 from an heterozygous bcl-2 male founder of the NSE73a line crossed with a female of the C57BL6/J strain (The Jackson Laboratory, Nossan, Milan, Italy); the colony was developed by selecting transgenic males from offspring and crossing them over with nontransgenic C57BL6/J females; bcl-2 females have a closed vagina and cannot be used for reproduction. The presence of the transgene was detected by PCR on DNA extracted from tail tissue of animals. The presence of the human Bcl-2 protein was occasionally revealed by immunohistochemistry on retinal tissue using the anti-human bcl-2 antibody from Dako (Milan, Italy) (clone 124); high density of retinal ganglion cells and large size of the optic nerves confirmed a typical bcl-2 phenotype. Mice used as controls were C57BL6/ $\mathrm{J}$ of the same litters but bcl-2-negative. A total number of 36 mice were used in this study; they ranged in age between 3 and 6 months.

Hybridoma cells. Mouse hybridoma cells producing IN-1 antibody (clone IN-1 C4) and control hybridoma cells producing antibodies against HRP (clone HRP 4HI) were used. Details about clone isolation and culturing conditions are given by Caroni and Schwab (1988). Before surgery, cells were thawed and resuspended at a concentration of 50,000 cells $/ \mathrm{ml}$ and grown in culture for $4-5 \mathrm{~d}$ in Iscove's medium supplemented with $6 \%$ fetal bovine serum, $2 \mathrm{~mm}$ L-glutamine, $100 \mathrm{U} / \mathrm{ml}$ penicillinstreptomycin, and $50 \mathrm{~mm} \beta$-mercaptoethanol. Before implantation, the supernatants of all the hybridoma cells used were tested for the production of mouse IgM (in the case of IN-1 C4) or IgG (in the case of HRP $4 \mathrm{HI}$ ), by using the assay Boehringer Mannheim (Milan, Italy) IsoStrip. IN-1 supernatant was also used to immunostain the NI 250 fraction of myelin in the intact mouse optic nerve and tract. The staining, revealed with anti-mouse FITC secondary antibodies (dilution of 1:200; Sigma, Milan, Italy), was very intense throughout the optic pathway.

Surgery. The animals were deeply anesthetized with Avertin $(1.2 \%$ tribromoethanol and $2.4 \%$ amylene hydrate in distilled water, $2 \mathrm{ml} / 100$ gm body weight), and the left optic nerve was crushed mechanically with thin surgical forceps. The crush was performed intracranially, $\sim 3 \mathrm{~mm}$ from the posterior pole of the eye; special care was taken to avoid mechanical damage to the retinal artery. We used three different experimental protocols: simple crush of the optic nerve (six wt and seven bcl-2 animals used); crush plus injection of IN-1 hybridoma cells (six wt and six bcl-2 used); crush of the optic nerve plus injection of HRP antibodies (four wt and three bcl-2 used).

Hybridoma injection. Six wt and six bcl-2 mice received an injection of IN-1, producing hybridoma cells directly into the lesioned nerve at the site of the crush operation. Approximately $3-5 \times 10^{5}$ cells in $1 \mathrm{ml}$ of HBSS were injected using a glass pipette pulled to a final tip of $20-30 \mu \mathrm{m}$ driven by an oil microinjector. Hybridoma cells were prestained with DiI ( $2 \mathrm{mg} / \mathrm{ml}$ in DMSO per milliliter of HBSS) to allow further recognition. Control experiments were performed injecting in the crushed nerves hybridoma cells producing antibody against HRP.

Histology. Four weeks after surgery, the left eyes of the operated animals were injected with $2 \mu \mathrm{l}$ of $20 \%$ neurobiotin tracer (Vector Laboratories, Burlingame, CA) in saline solution. One day after neurobiotin injection, the animals were perfused transcardially with $4 \%$ paraformaldehyde in $0.1 \mathrm{M}$ phosphate buffer; both optic nerves were carefully dissected, rinsed, cryoprotected with $30 \%$ sucrose, and flat frozen on a cryostat stage. Consecutive frozen sections were cut at $12 \mu \mathrm{m}$ thickness and reacted with 1:400 avidin-FITC to visualize neurobiotin. Adjacent sections were immunostained with antibodies against the $200 \mathrm{kDa}$ subunit of neurofilaments (clone N52; dilution of 1:200; Sigma) to allow visualization of the whole fiber population (Berry et al., 1996; Burne et al., 1996). Selected sections were immunostained using the F4/80 rat monoclonal antibody (dilution of 1:10; Serotec, Oxford, UK) specific for mouse microglia and macrophages (Austyn and Gordon, 1981). Secondary antibodies were tetramethylrhodamine isothiocyanate-anti-mouse $\operatorname{IgG}$ (Sigma, dilution 1:50) and Alexa 488-anti rat $\operatorname{IgG}$ (dilution of 1:400; Molecular Probes, Eugene, OR), respectively.

The whole series of sections obtained from each nerve was examined systematically with an Zeiss (Milan, Italy) Axioplan microscope equipped with epifluorescence. Representative sections were analyzed in detail with a Leica (Milan, Italy) TCS-NT confocal microscope. Confocal series were obtained through the whole section thickness $(10-12 \mu \mathrm{m})$; each focal series was visualized as a projection on single plane.

The optic nerves of two wt and two bcl-2 mice that had received a simple crush were post-fixed in $4 \%$ glutaraldehyde in $0.1 \mathrm{~m}$ phosphate buffer for $12 \mathrm{hr}$. Subsequently, each nerve was dissected transversally in four blocks, from the optic nerve head to the optic chiasm, which were processed separately; one block contained the optic nerve head and another the crush site, which was readily recognizable under the dissecting scope for its yellowish, transparent appearance. Nerve blocks were treated with $2 \%$ osmium tetroxide, stained with $1 \%$ uranyl acetate in maleate buffer, dehydrated in ethanol, and embedded in Epon-Araldite. Semithin sections, 1- to 2- $\mu \mathrm{m}$-thick, were cut with a diamond knife from the blocks containing the optic nerve heads and from those containing the lesion site, sectioned from its proximal side. Sections were stained with Epoxy Tissue Stain (Electron Microscopy Sciences, Fort Washington, PA) and examined with a Zeiss Axioplan photomicroscope.

Survival. The left retinas of three wt and three bcl-2 animals that had received crush of the left optic nerves 4 weeks before, plus the retinas of three wt that had received an injection of IN-1 hybridoma cells, were stained as whole mounts with $2 \mathrm{~mm}$ ethidium homodimer II (Molecular Probes) and examined at the confocal microscope. The total number of cells in the ganglion cell layer (GCL) was estimated from counting 16-20 regularly spaced $125 \times 250 \mu \mathrm{m}$ fields located in the four retinal quadrants and multiplying the average cellular densities to the retinal areas measured with an image analyzer (Imaging Inc., Ontario, Canada). The number of displaced amacrines and ganglion cells in the intact retinas of wt and bcl-2 mice were obtained from separate studies (Cenni et al., 1996; Jeon et al., 1998). Survival was estimated by subtracting the number of displaced amacrines from the total number of cells found in the GCL 1 month after surgery; the obtained values were expressed as a fraction of the number of GCs of the intact retinas.

Quantification of sprouting. A total of 20 nerves in which fibers were stained with neurobiotin were used to obtain a semiquantitative evaluation of axonal sprouting. Animals were distributed in the following groups: three wt mice that had received a simple crush (wt-crush); three bcl-2 mice with simple crush (bcl-2 crush); three wt mice with crush plus injection of HRP-hybridoma cells (wt HRP); three bcl-2 mice with crush plus HRP-hybridoma cells (bcl-2 HRP); three wt mice with crush plus IN-1-hybridoma cells (wt IN-1); and three bcl-2 mice with crush plus IN-1 hybridoma cells (bcl-2 IN-1). For each crushed nerve, representative sections were analyzed at the confocal microscope as described above. Images representing projections of nerve sections near the crush site were transferred on a microcomputer imaging device (Imaging, Inc.) M4 image analyzer. The intensity of fluorescence staining of fibers was determined by means of densitometric analysis at 10 different spots, spaced regularly along two selected lines perpendicular to the major axis of the nerve section. The first line corresponds to the border of the transected fibers (the virtual edge at which the majority of fibers stops); the second line is taken at a more proximal position (within a distance of $300 \mu \mathrm{m}$ from the first line, toward the optic nerve head). At each location, in each section, we calculated the ratio between the optical density (OD) measured at the first line and the OD at the second line, respectively. This value is referred to as ODR (optical density ratio).

\section{RESULTS}

\section{Effects of crush lesion in wt and bcl-2 mice 1 month after surgery}

Figure 1 reports the fractions of surviving retinal ganglion cells in adult wt and bcl-2 mice (three retinas for each strain) 1 month after intracranial crush of the optic nerve. In the wt, optic nerve crush results in the survival of approximately half retinal ganglion cells $(44 \%)$. On the other hand, in the bcl-2 mouse, virtually $100 \%$ of RGCs are still alive. This confirms the powerful survival-promoting effect of the bcl-2 gene.

Figure 2 shows the effects of crush lesion onto optic fiber preservation in wt and bcl-2 optic nerves in transversal semithin sections (two nerves examined for each strain). Both optic nerves are illustrated at the emergence from the posterior pole of the eye and at the crush site, respectively. At the optic nerve head, individual degenerating fibers are visible: however, the overall nerve structure is still well preserved, especially in the bcl-2 mouse (Fig. 2A-D). Toward the crush site, fibers with disorganized morphology become more evident, in both the wt and the bcl-2 nerve (Fig. 2E,F). Axons have retracted and a central core of vacuolization, and tissue reorganization has appeared. Higher magnification of the lesion site shows individual dying fibers all over the whole nerve surface; in the bcl-2, normal fibers are still 


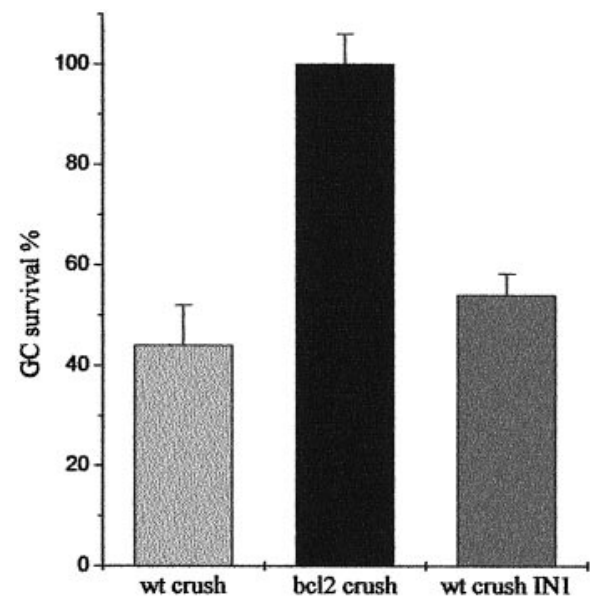

Figure 1. Survival rate of retinal ganglion cells 1 month after intracranial crush of the left optic nerve. Columns are percentage average survivals with error bars. The first column refers to wild-type retinas $(n=3)$ and the second column to bcl-2 retinas $(n=3)$. Notice that survival is $100 \%$ in bcl-2 animals. The last column shows the survival rate of ganglion cells in wt retinas $(n=3)$ after injection of IN-1 hybridoma cells at the site of crush.

visible, mostly in the superficial ring of the nerve (Fig. 2G,H). When crushed optic nerves are analyzed in longitudinal sections after neurobiotin injection in the corresponding eyes, individual fibers are visualized along their course to the lesion point (Fig. 3). After crush, fibers end at different positions along the longitudinal axis of the nerve, depending on the variable extent of retraction. We define as "border of the fibers" the line (perpendicular to the longitudinal axis of the nerve) at which the majority of axons stops. This line represents one of the boundaries we used for densitometric analysis (see below). It is important to point out that, 1 month after crush, there is no spontaneous sprouting of the fibers or elongation beyond the border, in both the wt and bcl-2 nerves (four nerves examined in longitudinal serial sections for each strain of mice).

\section{Effects of IN-1 hybridoma cell injections}

To create a favorable environment to optic fiber regeneration, we injected in the optic nerves hybridoma cells producing an antibody (IN-1) known to bind and neutralize a powerful inhibitor of fiber elongation associated with CNS myelin (NI-250).

Injection of $1 \mu \mathrm{l}$ of suspension containing $3-5 \times 10^{5}$ cells was performed in the crushed optic nerves of mice. The supernatant of IN-1 hybridoma cells was tested for the production of mouse IgM before each injection. The actual number of cells injected can be expected to vary to some extent, because some of the suspension was observed to flow out from the nerve after pipette withdrawal. The successful outcome of the injection was tested at the time of nerve dissection; hybridoma cells, labeled with DiI could be visualized with a fluorescence microscope in the intact nerves and are visible in Figures 4 and 5 in longitudinal nerve sections. One month after crush, hybridoma cells appeared mostly concentrated near the injection site without remarkable migration inside the nerve. To check whether IN-1 hybridoma injection could exert some effect in preventing neuronal cell death, we computed the number of resilient ganglion cell in the retinas of three wt mice treated with IN-1 antibody. In these animals, RGC survival was in the same range $(54 \%)$ observed in the case of $\mathrm{wt}$ mice with a simple crush lesion (Fig. 1). Consequently, in our system, we can exclude a role of IN-1-producing hybridoma cells in affecting neuronal survival.

The effects of IN-1 on crushed fibers were evaluated after systematic examination of longitudinal sections of optic nerves after neurobiotin injection in the corresponding eyes. In both wt and bcl-2 (six animals for each strain), we observed that the majority of fibers that reached the crush site interrupted abruptly (Fig. $4 A, B$ ). Only in the case of one bcl-2, fibers were seen to regrow for $\sim 500 \mu \mathrm{m}$ beyond the major border of fibers. Elongation occurred in axons running at the surface of the optic nerve. In all the remaining cases, fibers did not succeed in growing more than 10-20 $\mu \mathrm{m}$. We can conclude that, despite of the administration of IN-1 hybridoma cells in the lesion area, we did not observe any significant regeneration.

On the other hand, we observed a sprouting promoting effect after injection of IN-1 hybridoma cells; in all bcl-2 mice, we found fibers that sprouted at the lesion site. Figure $5 \mathrm{~A}$ shows the longitudinal section of one of these nerves in which sprouting is particularly profuse. Fibers appear green, and DiI-stained hybridoma cells are red. Many axon terminals can be seen at the site of crush (indicated by arrows); some of them show large size and intense staining, as is typical of degenerating fibers, although the majority are thin and sprout profusely. Sprouting processes have often a tortuous course with ramifications. Similar sprouting profiles, in variable number and degree of complexity, could be observed in all the IN-1-treated bcl-2 mice. Immunocytochemistry with an antibody against the heavy subunit of neurofilaments showed that large-sized degenerating fibers were intensely stained, whereas the thinner ones, exhibiting a tortuous course revealed by neurobiotin injection, were usually not labeled. The absence of neurofilament staining in processes with morphology typical of newly formed fibers confirms that they are sprouting axonal terminals. It has been shown that the heavy subunit of neurofilaments is absent from the tips of actively growing axons (Foster et al., 1987).

Figure $4 B$ also shows the result of the injection of IN-1producing hybridoma cells in crushed nerves of wt mice. As can be observed at high magnification (Fig. $5 B$ ), most of the axons in the wt end freely at the border of fibers, without obvious ramifications. Retraction is evident. A quantitative analysis of fiber behavior in various experimental groups is illustrated later and includes an evaluation of the sprouting effect.

\section{Control experiments}

Control experiments were performed injecting in the crushed nerves of four bcl-2 and three wild-type mice hybridoma cells producing antibodies against HRP; cell injections followed the same protocol used for IN-1 hybridoma cells. Longitudinal sections of the nerves were examined systematically after neurobiotin injections in the eyes. Results are shown in Figure 6. Axons behaved mostly as in the case of a simple crush; namely, they stopped at the lesion site without sprouting or elongating, in both wt and bcl-2 animals. Retraction at the lesion site was again visible. We concluded that the injection of hybridoma cells that do not release antibodies interfering with axonal elongation is not sufficient per se to promote sprouting in bcl-2 mice.

In addition, we looked for major differences between wt and bcl-2 in the macrophage-microglia response to crush after hybridoma cell injection. Figure 7 shows F4/80 staining in crushed nerves of wt and bcl-2 that had received an injection of HRP cells. 

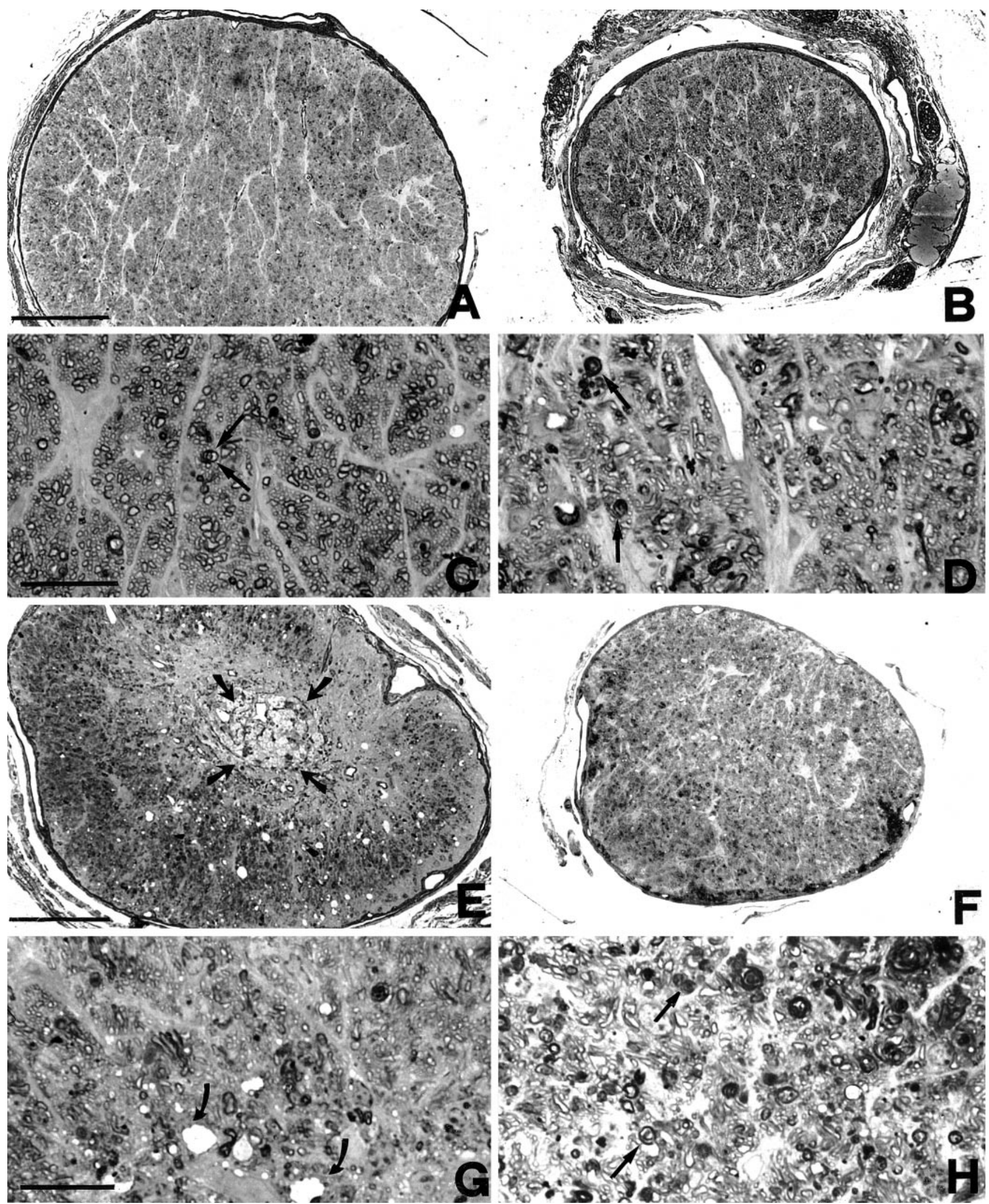

Figure 2. Light micrographs of the optic nerves of bcl-2 (left) and wild-type (right) mice 1 month after intracranial crush. $A, B$, Sections obtained in the proximity of the optic nerve head. $C, D$, High magnifications of $A$ and $B$, respectively. The general morphology is well preserved in both wt and bcl-2 $(A, B)$; degenerating fibers, characterized by a disorganized myelin sheath, are rare in the bcl-2 and more abundant in the wt (arrows in $C$ and $D$ ). $E$, $F$, Sections obtained near the crush site and proximal to it. At low magnification, a central core of degeneration is evident in the bcl-2 nerve (arrows in $E$ ). At higher magnification, vacuoles are visible in the nerve core of the bcl-2 (arrows in $G$ ); however, numerous fibers appear still viable at more superficial positions (top of $G$ ). In the nerve of the wt $(F, H)$ degeneration of fibers is widespread, as clearly illustrated in $H$; arrows point to altered myelin. Scale bars: (in $A, E) A, B, E, F, 100 \mu \mathrm{m}$; (in $C, G) C, D, G, H, 20 \mu \mathrm{m}$. 

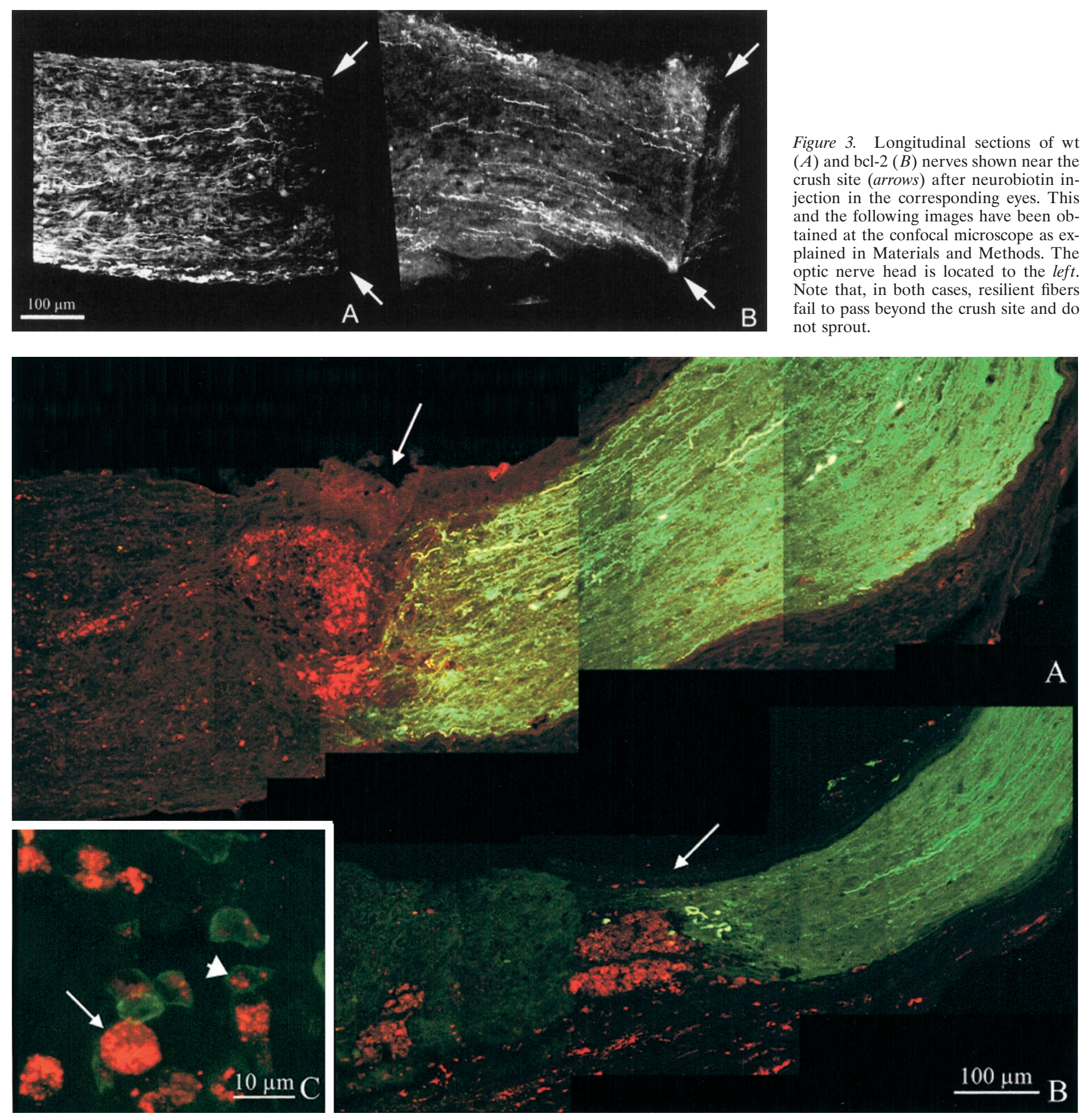

Figure 4. Longitudinal sections of crushed optic nerves of bcl-2 $(A)$ and wt $(B)$ mice 1 month after ON crush and implantation of IN-1 hybridoma cells. Fibers are labeled green by neurobiotin injected into the eye and revealed with FITC-avidin. Hybridoma cells, stained with DiI, appear red. Arrows point to the crush site. $C$, Staining with F4/80 antibody, specific for mouse microglia and macrophages. Double-labeled cells (short arrow) are immunopositive cells that have engulfed DiI, and the red ones are hybridoma cells (long arrow).

The staining revealed numerous cells, identifiable as macrophages because of their large size, scattered all over the nerves, although mostly concentrated at the lesion site. They stained so intensely that small-sized microglial cells were primarily obscured. F4/80-positive cells of similar morphology were absent from the contralateral, intact nerves in which only microglia was labeled. There were no evident differences between wt and bcl-2 mice in the F4/80 pattern of immunoreactivity. Thus, we can expect that macrophage-microglia recruitment follows similar rules in the two cases. However, this issue can be clarified only after a systematic analysis of the immune response after optic nerve crush in the two strains of mice, similar to the study of Lawson et al. (1994). Because this was beyond the scope of this study, we did not make any attempt to go into further details. 


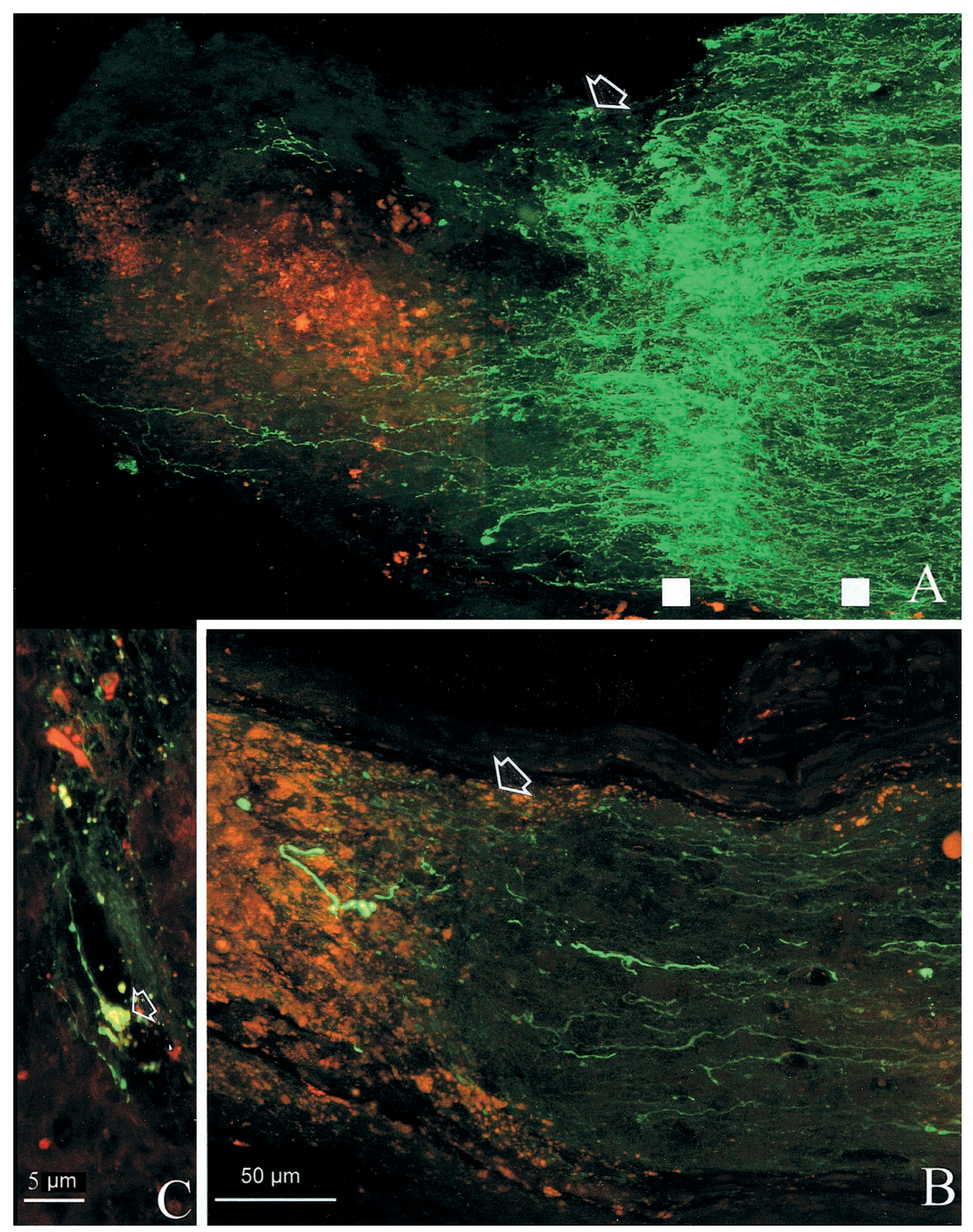

Figure 5. High magnification of bcl-2 $(A)$ and wt $(B)$ nerves illustrated at the site of crush. Arrows indicate the lesion site. Note the wealth of sprouting profiles in the bcl-2 $(A)$ compared with the paucity of fibers present in the wild type $(B)$. White squares are examples of the points intersected by lines perpendicular to the nerve major axes and used to calculate the density values of fluorescence staining. The square on the left is at the sprouting site, and the right one is located more proximally. $C$, The tip of a regenerating fiber from $A$ exhibits the typical morphology of a growth cone (arrow).

\section{Semiquantitative evaluation of fiber sprouting}

We tried to express quantitatively the extent of sprouting, performing OD measures on longitudinal sections of those nerves in which the fibers could be visualized clearly after neurobiotin injection. We calculated the ODR between OD at the border of fibers and OD at a more proximal position, within a maximum distance of $300 \mu \mathrm{m}$ (see Materials and Methods). ODR values greater than one are indicative of an increase in the number of fibers along the major axis of the nerve and thus are suggestive of sprouting. ODR values were calculated for all the six experimental groups: wt crush, bcl-2 crush, wt HRP, bcl-2 HRP, wt IN-1, and bcl-2 IN-1. Results are summarized in Figure 8. 


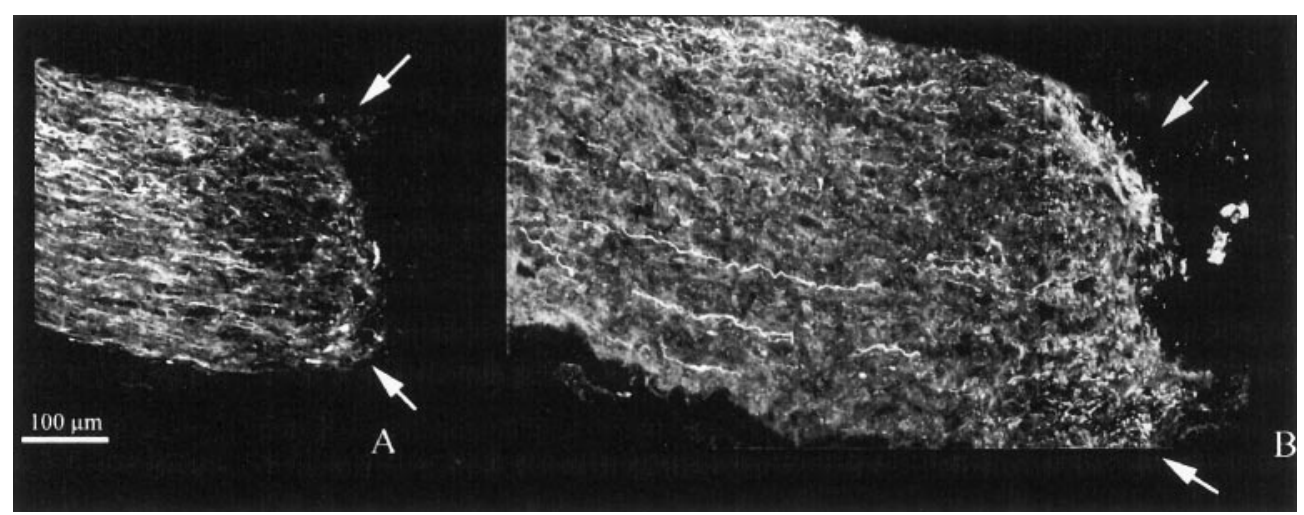

Figure 6. Longitudinal sections of $\mathrm{wt}$ $(A)$ and bcl-2 $(B)$ nerves that have received an injection of HRP hybridoma cells at the site of crush. Fibers stop at the lesion site, indicated by arrows.

In the wt crush group, ODR was $0.87 \pm 0.07$, and it was not significantly different from that of the bcl-2 crush group (0.99 \pm 0.05; $p=0.06$; Mann-Whitney rank sum test).

ODR values among the three groups of wt mice (wt crush, wt HRP, and wt IN-1) were also very similar and not statistically different ( $p=0.076$; Kruskal-Wallis one-way ANOVA). In contrast, in the three groups of bcl-2 animals (bcl-2 crush, bcl-2 HRP, and bcl-2 IN-1), ODR values were statistically different ( $p=$ 0.001; one-way ANOVA on ranks). To isolate the groups that differed from the others, we performed a post hoc group-to-group comparison using the Mann-Whitney rank sum test. We obtained that there was no significant difference between bcl-2 HRP and bcl-2 crush $(p=0.451)$, whereas bcl-2 IN-1 differed from both bcl-2 crush and bcl-2 HRP ( $p=0.001$ in both cases). Thus, densitometric analysis confirms that sprouting is promoted only in bcl-2 animals treated with IN-1.

\section{DISCUSSION}

The aim of this study was to evaluate the capabilities of adult RGCs overexpressing the anti-apoptotic protein bcl-2 to regenerate their injured axons within an environment made more permissive to axonal elongation.

To test such potentials in an in vivo model, we made use of bcl-2 transgenic mice treated with $\mathrm{IN}-1$ antibodies.

In previous work (Cenni et al., 1996), we described the powerful effect of bcl-2 overexpression in rescuing RGCs from death induced by intracranial optic nerve cut. Whereas only $5 \%$ RGCs survived in wt mouse 2 months after the lesion, 63\% RGCs were still alive and functional 3 months after the lesion (Porciatti et al., 1996) in the transgenic animal.

In the present study, an intracranial crush instead of a transection of the optic nerve was performed. Crush was adopted because it allows axotomy of RGCs while ensuring the preservation of the optic nerve connective sheaths, which can act as a scaffold for possibly regenerating fibers.

First, we estimated the number of RGCs surviving optic nerve crush in the bcl-2 and in the wt. Similar to what observed after optic nerve transection, a large difference between bcl-2 and wt mice was found; 1 month after surgery, virtually the whole population of retinal ganglion cells survived in the bcl-2, whereas $44 \%$ remained in the wt retina.

The RGC survival rate that we have estimated in the adult wt mouse 1 month after crush is inferior to the $80 \%$ value estimated for the adult rat, at the same time and after the same lesion (Berkelaar et al., 1994). In the mouse, the intracranial crush of the optic nerve produces shorter proximal axonal stumps compared with the rat. Because resilient axonal segments can be considered as a source of factors promoting the survival of retinal ganglion cells, it can be postulated that, in our experimental conditions, axotomized RGCs can benefit from a more limited supply of trophic molecules compared with RGCs of the rat.

We studied the morphology of the axotomized fibers in both transversal and longitudinal sections. At the optic nerve head, most of the fibers were well preserved, especially in bcl-2 mice. At more distal positions, very close to the lesion area, morphology was profoundly affected, and the number of degenerating profiles increased evidently. So, fibers retracted from the immediate proximity of the site of crush in both wt and bcl-2.

Compared with the results reported in previous work (Cenni et al., 1996; Chierzi et al., 1998), these observations show that the difference between wt and bcl-2 is less pronounced after crush than after cut of the optic nerve. Actually, previous work refers to the effects of optic nerve cut 2-3 months after surgery. At this time, the optic nerve head of the wt contains so few fibers that the difference with bcl-2 is striking. Such protective effects of bcl-2 appear less dramatic 1 month after crush, when degeneration of both cell bodies and fibers in the wt is still moderate. Hence, the present results are not readily comparable with those of the previous work for two reasons: (1) here, we performed a crush instead of a cut of the optic nerve; and (2) we choose a survival time of 1 month to limit loss of antibody production by transplanted hybridoma cells.

Two main conclusions emerge clearly from our data. The first is that regeneration in the CNS is not substantially facilitated in the IN-1-treated bcl-2 animals in which only sprouting appears to be definitely more abundant than in wild-type controls.

This conclusion is unfortunately negative and somewhat contrary to expectations in that the absence of degeneration of RGCs after crush of the optic nerve and the block of myelin-derived inhibitory factors through the administration of IN-1 fulfilled at least two necessary conditions for regeneration to occur.

The second conclusion concerns the relevance that the hostility of the CNS to regeneration plays in an in vivo system; experiments in vitro have given evidence that bcl-2 overexpressing neurons can indeed regenerate over a permissive substrate (Chen et al., 1997). Conclusions or generalizations from the in vitro experiments could be somehow hazardous and misleading when applied to the in vivo condition.

Our results show that the administration of IN-1 in both the wild-type and the bcl-2 mouse optic nerve is not sufficient to allow significant regeneration of transected fibers beyond the site of crush. Injection of IN-1-producing hybridoma cells on the 


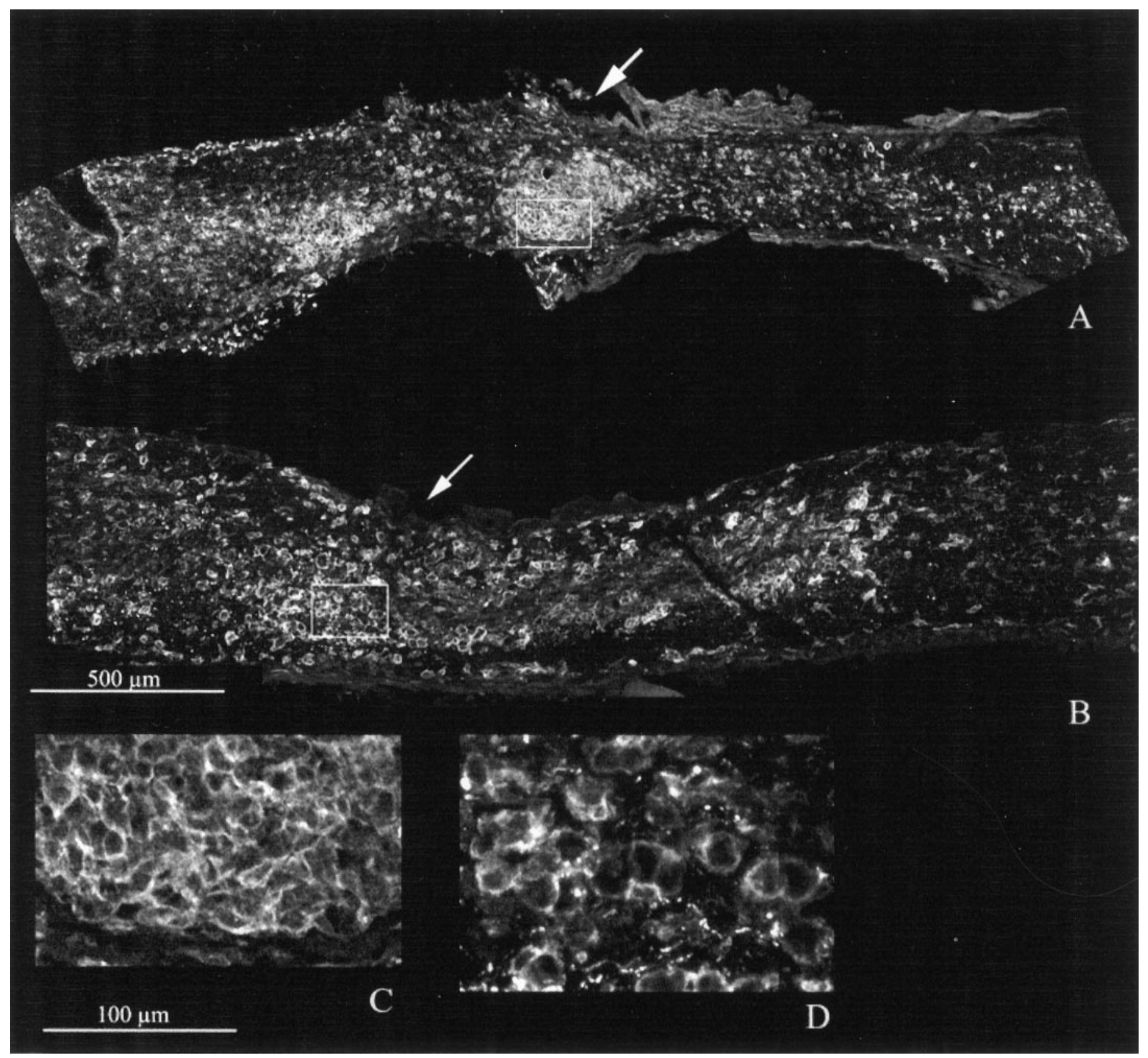

Figure 7. F4/80 staining in crushed nerves of wt $(A)$ and bcl-2 $(B)$ that had received an injection of HRP cells. The crush site is indicated by arrows. Stained cells are dense at the lesion site; $C$ and $D$ (enlargements of the fields shown as rectangles in $A$ and $B$ ) illustrate large size cells having a round morphology, typical of macrophages. Notice that there are not obvious differences in the staining between $A$ and $B$.

crushed nerve has been used by Weibel et al. (1994) to induce regeneration of transected RGC axons. They implanted IN-1producing hybridoma cells over the crushed optic nerves of rats that had received FGF into the vitreous and observed longdistance regeneration of few axotomized fibers. However, the experimental design of Weibel et al. differs from the one used in the present study in some relevant aspects; they injected fibroblast growth factor to prevent RGC death and axonal degeneration, whereas in our experimental protocol, both these effects were prevented by overexpression of bcl-2. Furthermore, the animals used were relatively immature (16- to 18-d-old), whereas we used adult mice. This difference might be relevant in the amount of regeneration occurring afterward, because it is well known that lesioned fibers of the immature CNS show a more pronounced attitude to sprouting and regeneration compared with fibers of the mature CNS (Bates and Stelzner, 1993).

The possibility exists that the concentration reached by the IN-1 antibody was not high enough to counteract the inhibitory action of myelin, although we used relatively large amounts of hybridoma cells directly at the crush site. It is well known that even very low concentrations of myelin can arrest growth cone progression in vitro. Because IN-1 hybridoma cells did not migrate in the distal stump of the optic nerves in which degenerating myelin persists for a long time, we can be confident that the effects of myelin inhibitors have been counteracted within the immediate proximity of the crush site. Indeed, this is confirmed by the 

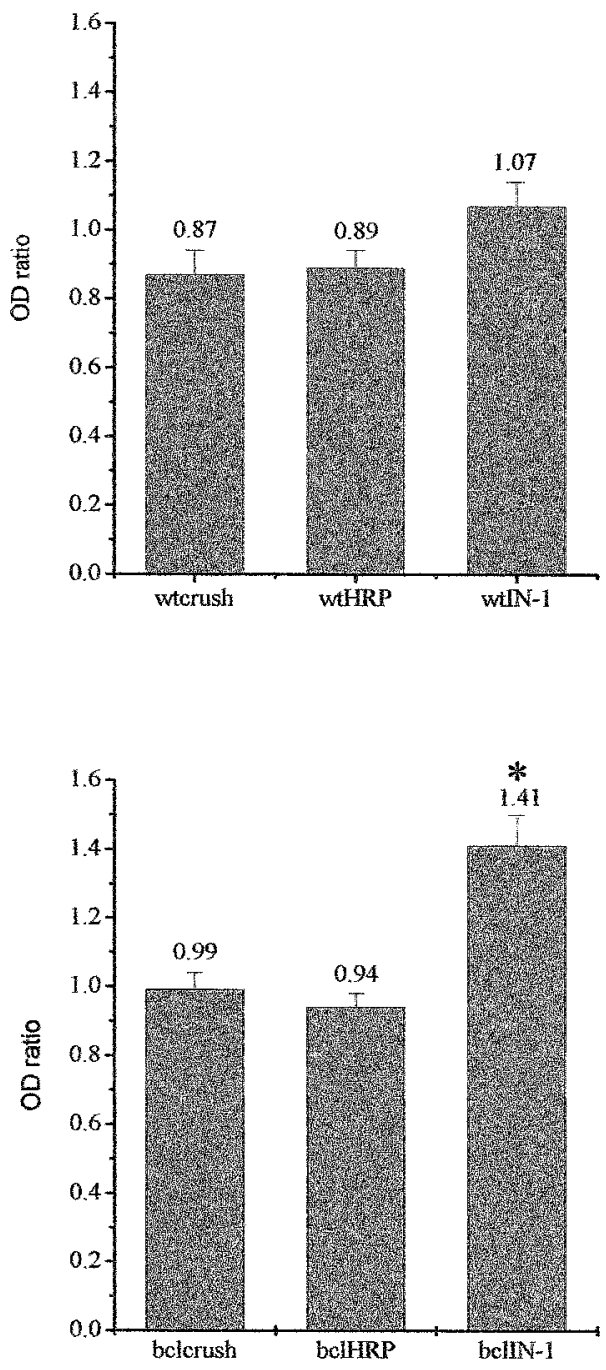

Figure 8. Semiquantitative analysis of fiber responses in different experimental conditions. Top shows the wt groups; bottom shows the bcl-2. Columns represent average OD ratios with standard errors. The asterisk points out the significant difference among the bcl-2 IN-1 group and the remaining two.

observation that sprouting was promoted by $\mathrm{IN}-1$ hybridoma cells in bcl-2.

In addition, the failure of the majority of axons to regenerate in the IN-1-treated bcl-2 mice can be explained by postulating that other inhibitory factors in addition to myelin proteins are present at the lesion site.

In particular, the formation of a scar, a process involving different cell types of the nervous and immune systems (activated astrocytes, activated microglia, meningeal cells, and inflammatory macrophages and leukocytes) is believed to play an important role in creating a hostile environment to regeneration. Indeed, we observed macrophage concentration at the crush site after hybridoma injection, in both wt and bcl-2. In the crushed optic nerve, the scar is extending across the whole nerve thickness; regenerating fibers have to grow through a region of greatly modified and rearranged tissue, because no bridges of intact optic nerve remain for their passage. Different lines of evidence have pointed to reactive astrocytes of the scar as powerful elements impeding axonal regrowth (McKeon et al., 1995; Davies et al., 1997). Reaction of astrocytes to injury results in morphological hypertrophy and in the synthesis and secretion in the extracellular matrix of inhibitory molecules, such as the proteoglycan chondroitin sulfate (McKeon et al., 1995). Reactive astrocytes taken from the crushed optic nerves of rats represent a nonpermissive substrate for the elongation of CNS axons (Bähr et al., 1995).

Even if in our experimental model the axons failed to elongate beyond the crush site, in bcl-2 mice injected with IN-1-producing hybridoma cells, we observed axonal sprouting at the border of transected fibers. We tried to express quantitatively the extent of sprouting observed in the different experimental cases calculating optical density ratios at the lesion site for different experimental protocols. We find that, in wt animals, no treatment is able to increase significantly ODRs, whereas in bcl-2 mice, the injection of IN-1-producing hybridoma cells induces a significant increase in ODRs, indicating an effect in promoting sprouting.

It appears that the administration of IN-1 antibodies to the crushed optic nerve can unmask an effect of bcl-2 in eliciting the sprouting of transected fibers.

These results suggest a correlation between bcl-2 overexpression and axonal growth in an adult animal. Such effect of bcl-2, distinct from the well known survival effect, is in agreement with some recent findings: embryonic retinal ganglion cells (embryonic day 18) from bcl-2 mice have been shown to retain elongation capabilities typical of earlier stages of development, which are absent in RGCs of wt mice of the same age (Chen et al., 1997). In addition, neurons transfected with the bcl-2 gene show an increase in the outgrowth of neuritic processes (Zhang et al., 1996; Middleton et al., 1998). Our results also correlate with recent studies on the expression of the endogenous bcl-2 gene in primate brain: the Bcl-2 protein, expressed diffusely during brain development, becomes restricted in adult life to distinct structures of the CNS, characterized by persistent plasticity (Bernier and Parent, 1998).

Although the mechanisms of action of bcl-2 are not well clarified, some hypothesis can be formulated to explain a possible permissive action of the transgene overexpression on axonal sprouting, in the presence of IN-1. It is known that the effect of the CNS myelin protein NI 250 (inhibited by IN-1 antibodies) in mediating growth cone collapse is associated with a large increase in cytosolic calcium released from intracellular stores. The administration of IN-1 antibodies inhibits growth cone collapse and prevents the increase in cytosolic calcium (Bandtlow et al., 1993). The Bcl-2 protein is involved in the control of the calcium homeostasis as well; the protein, localized on the membrane of intracellular organelles, such as endoplasmic reticulum, nucleus, and mitochondria, is capable of preventing $\mathrm{Ca}$ efflux from such stores to the cytoplasm in response to apoptotic stimuli (Lam, 1994; Guo et al., 1997; Miller, 1998). Thus, it is conceivable that IN-1 administration and bcl-2 overexpression can act synergistically, both limiting the increase of calcium levels associated with growth cone arrest. When IN-1 antibodies alleviate the strong inhibition exerted by myelin, bcl-2 fibers are able to sprout.

\section{REFERENCES}

Adams JM, Cory S (1998) The bcl-2 protein family: arbiters of cell survival. Science 281:1322-1326.

Allsopp TE, Wyatt S, Patterson HF, Davies AM (1993) The protooncogene bcl-2 can selectively rescue neurotrophic factor-dependent neurons from apoptosis. Cell 73:295-307.

Austyn JM, Gordon S (1981) F4/80, a monoclonal antibody directed specifically against the mouse macrophage. Eur J Immunol 11:805-815.

Bähr M, Przyrembel C, Bastmeyer M (1995) Astrocytes from adult rat optic nerves are nonpermissive for regenerating retinal ganglion cell axons. Exp Neurol 131:211-220. 
Bandtlow CE, Schmidt MF, Hassinger TD, Schwab ME, Kater SB (1993) Role of intracellular calcium in NI-35-evoked collapse of neuronal growth cones. Science 259:80-83.

Bates CA, Stelzner DJ (1993) Extension and regeneration of corticospinal axons after early spinal injury and the maintenance of corticospinal topography. Exp Neurol 123:106-117.

Berkelaar M, Clarke DB, Wang Y-C, Bray GM, Aguayo AJ (1994) Axotomy results in delayed death and apoptosis of retinal ganglion cells in adult rats. J Neurosci 14:4368-4374.

Bernier PJ, Parent A (1998) Bcl-2 protein as a marker of neuronal immaturity in postnatal primate brain. J Neurosci 18: 2486-2497.

Berry M, Carlile J, Hunter H (1996) Peripheral nerve explants grafted into the vitreous body of the eye promote the regeneration of retinal ganglion cell axons severed in the optic nerve. J Neurocytol 25:147-170.

Bonfanti L, Strettoi E, Chierzi S, Cenni MC, Liu X-H, Martinou J-C, Maffei L, Rabacchi SA (1996) Protection of retinal ganglion cells from natural and axotomy-induced cell death in neonatal transgenic mice overexpressing bcl-2. J Neurosci 16:4186-4194.

Brösamle C, Schwab ME (1996) Axonal regeneration in the mammalian CNS. Semin Neurosci 8:107-113.

Burne JF, Staple JK, Raff M (1996) Glial cells are increased proportionally in transgenic optic nerves with increased numbers of axons. J Neurosci 16:2064-2073.

Carmignoto G, Maffei L, Candeo P, Canella R, Comelli C (1989) Effects of NGF on the survival of rat retinal ganglion cells following optic nerve section. J Neurosci 9:1263-1272.

Caroni P, Schwab ME (1988) Antibody against myelin-associated inhibitor of neurite growth neutralizes nonpermissive substrate properties of CNS white matter. Neuron 1:85-96.

Cenni MC, Bonfanti L, Martinou J-C, Ratto GM, Strettoi E, Maffei L (1996) Long-term survival of retinal ganglion cells following optic nerve section in adult bcl-2 transgenic mice. Eur $\mathrm{J}$ Neurosci 8:1735-1745.

Chen DF, Schneider GE, Martinou J-C, Tonegawa S (1997) bcl-2 promotes regeneration of severed axons in mammalian CNS. Nature 385:434-439.

Chierzi S, Cenni MC, Maffei L, Pizzorusso T, Porciatti V, Ratto GM, Strettoi E (1998) Protection of retinal ganglion cells and preservation of function after optic nerve lesion in bcl-2 transgenic mice. Vision Res 38:1537-1543.

Davies SJA, Fitch MT, Memberg SP, Hall AK, Raisman G, Silver J (1997) Regeneration of adult axons in white matter tracts of the central nervous system. Nature 390:680-683.

Foster GA, Dahl D, Lee VMY (1987) Temporal and topographic relationship between the phosphorylated and nonphosphorylated epitopes of the $200 \mathrm{kDa}$ neurofilament protein during development in vitro. J Neurosci 7:2651-2663.

Guo Q, Sopher BL, Furukawa K, Pham DG, Robinson N, Martin GM, Mattson MP (1997) Alzheimer's presenilin mutation sensitizes neural cells to apoptosis induced by trophic factor withdrawal and amyloid peptide: involvement of calcium and oxyradicals. J Neurosci 17:4212-4222.

Hilton M, Middleton G, Davies AM (1997) Bcl-2 influences axonal growth rate in embryonic sensory neurons. Curr Biol 7:798-800.
Jeon CJ, Strettoi E, Masland RH (1998) the major cell populations of the mouse retina. J Neurosci 18:8936-8946.

Lam M, Dubyak G, Chen L, Nunez G, Miesfeld RL, Distelhorst CW (1994) Evidence that Bcl-2 represses apoptosis by regulating endoplasmic reticulum-associated $\mathrm{Ca}^{2+}$ fluxes. Proc Natl Acad Sci USA 91:6569-6573.

Lawson LJ, Frost L, Risbridger J, Fearn S, Perry VH (1994) Quantification of the mononuclear phagocyte response to Wallerian degeneration of the optic nerve. J Neurocytol 23:729-744.

Maffei L, Carmignoto G, Perry VH, Candeo P, Ferrari G (1990) Schwann cells promote the survival of rat retinal ganglion cells after optic nerve section. Proc Natl Acad Sci USA 87:1855-1859.

Mansour-Robaey S, Clarke DB, Wang YC, Bray GM, Aguayo AJ (1994) Effects of ocular injury and administration of brain-derived neurotrophic factor on survival and regrowth of axotomized retinal ganglion cells. Proc Natl Acad Sci USA 91:1632-1636.

Martinou J-C, Dubois-Dauphin M, Staple J, Rodriguez I, Frankowski H, Missoten M, Albertini P, Talabot D, Catsicas S, Pietra C, Huarte J (1994) Overexpression of bcl-2 in transgenic mice protects neurons from naturally occurring cell death and experimental ischemia. Neuron 13:1017-1030.

McKeon RJ, Hoke A, Silver J (1995) Injury-induced proteoglycans inhibit the potential for laminin-mediated axon growth on astrocytic scars. Exp Neurol 136:32-43.

Middleton G, Pin LGP, Wyatt S, Davies AM (1998) Bcl-2 accelerates the maturation of early sensory neurons. J Neurosci 18:3344-3350.

Miller RJ (1998) Mitochondria—the Kraken wakes! Trends Neurosci 21:95-97.

Porciatti V, Pizzorusso T, Cenni MC, Maffei L (1996) The visual response of retinal ganglion cells is not altered by optic nerve transection in transgenic mice overexpressing bcl-2. Proc Natl Acad Sci USA 93:14955-14959.

Thanos S, Bähr M, Barde Y-A, Vanselow J (1989) Survival and axonal elongation of adult retinal ganglion cells. In vitro effects of lesioned sciatic nerve and brain-derived neurotrophic factor. Eur J Neurosci $1: 19-26$.

Vidal-Sanz M, Bray GM, Villegas-Perez MP, Thanos S, Aguayo AT (1987) Axonal regeneration and synapse formation in the superior colliculus by retinal ganglion cells in the adult rat. J Neurosci 7:2894-2909.

Villegas-Perez MP, Vidal-Sanz M, Bray GM, Aguayo AJ (1988) Influences of peripheral nerve grafts on the survival and regrowth of axotomized retinal ganglion cells in adult rats. J Neurosci 8:265-280.

Weibel D, Cadelli D, Schwab ME (1994) Regeneration of lesioned rat optic nerve fibers is improved after neutralization of myelin-associated neurite growth inhibitors. Brain Res 642:259-266.

Zhang KZ, Westberg JA, Holtta E, Andersson LC (1996) Bcl-2 regulates neuronal differentiation. Proc Natl Acad Sci USA 93:4504-4508.

Zhong LT, Sarafian T, Kane D, Charles A, Mah S, Edwards R, Bredsen D (1993) Bcl-2 inhibits death of central neural cells induced by multiple agents. Proc Natl Acad Sci USA 90:4533-4537. 